\title{
METABOLISM OF TRYPTOPHAN IN THE LIVER: INTERFERENCE WITH DECARBOXYLATION OF OTHER AROMATIC AMINO ACIDS
}

\begin{abstract}
Jaroslav Dršata ${ }^{1}$ and Eliška Marklováa ${ }^{2}$
Charles University in Prague, Faculty of Pharmacy: Department of Biochemical Sciences ${ }^{1}$; Charles University in Prague, Faculty of Medicine in Hradec Králové: Department of Pediatrics ${ }^{2}$

Summary: Decarboxylation of aromatic amino acid in mammalian tissues is catalyzed by aromatic amino acid decarboxylase (EC. 4.1.1.28, AAD). The enzyme differs in its affinity to individual aromatic amino acids, the best substrates being 3,4-dihydroxyphenylalanine (dopa) and 5-hydroxytryptophan. Surprisingly, AAD is abundant in the liver, where the substrates with rather low affinity to AAD as tryptophan, phenylalanine, and tyrosine are offered to decarboxylation. In the present paper, the possibility of interference of tryptophan with decarboxylation of phenylalanine, tyrosine as well as dopa in the liver was investigated. The AAD activity was measured radiometrically with $1-{ }^{14} \mathrm{C}$ - labeled aromatic amino acid substrates using the rat liver enzyme. The influence of tryptophan on decarboxylation of tyrosine was formally competitive with $K_{i}=9.2 \times 10^{-3} \mathrm{M}$, while the inhibition of decarboxylation of phenylalanine by tryptophan was non-competitive with $K_{i}$ at $2.75 \times 10^{-2} \mathrm{M}$. The effect of tryptophan on decarboxylation of dopa was small and it could not be expressed in terms of inhibition kinetics and inhibition constant. At physiological concentrations of aromatic amino acids in plasma, tryptophan does not seem to have remarkable effects on decarboxylation of phenylalanine, tyrosine, and dopa in the liver.
\end{abstract}

Key words: Metabolism of tryptophan; Aromatic amino acid decarboxylase; Tyrosine decarboxylation; Phenylalanine decarboxylation; Inhibition; Liver

\section{Introduction}

Tryptophan may be metabolized by several different pathways. The major quantitative breakdown route via kynurenine leads to nicotinic acid and to the synthesis of the NAD coenzyme, other pathways involve indolic compounds. The hydroxyindole pathway of tryptophan metabolism via decarboxylation of 5-hydroxytryptophan provides the neurotransmitter serotonin, and the hormone melatonin in the pineal gland. The other pathways partly participate in the indolylacetic acid and the speculative indolylacrylic acid formation $(1,2)$.

As for the decarboxylation step in aromatic amino acid metabolism, it is catalyzed by aromatic amino acid decarboxylase (EC. 4.1.1.28, AAD), an enzyme with broad substrate specificity. Many years ago, catalyzed decarboxylation of L-3,4-dihydroxyphenylalanine (dopa) and 5-hydroxytryptophan was found out in mammalian kidney and liver extracts $(3,4)$. Lovenberg et al. (5) found AAD to have affinity also to other aromatic amino acids. Among them, decarboxylation of tryptophan is considered to be a quantitatively significant route of metabolism of this amino acid and decarboxylation of tryptophan increases according to its intake (6). Owing to the high substrate affinity of L-3,4dihydroxyphenylalanine and 5-hydroxytryptophan to AAD, an important role of this enzyme in the synthesis of cate- cholamines including dopamine, noradrenaline and adrenaline, and in the creation of serotonin is evident. Affinity of the other aromatic amino acids to AAD is comparatively much lower. In fact, neither L-3,4-dihydroxyphenylalanine nor 5-hydroxytryptophan are the compounds that are offered to the liver at higher concentrations, in comparison to more common amino acids as phenylalanine, tyrosine, and tryptophan $(7,8)$. For this reason it is rather surprising that the enzyme is particularly abundant in the liver, where creation of its main products under physiological conditions is low, and the significance of the enzyme is not apparent so far.

Since the same enzyme catalyzes decarboxylation of a group of amino acids with similar but not identical structures, the effect of various analogues of these compounds have been studied for years. The aim of those studies was to affect the synthesis of biologically active decarboxylation products of aromatic amino acids. We have investigated mammalian AAD activity with the substrate L-3,4-dihydroxyphenylalanine under various conditions $(9,10)$. Other authors (11) studied the in vitro effect of analogues of phenylalanine and tryptophan on the rat brain enzyme kinetics with the same substrate. Bosin et al. (12) examined the influence of tryptophan analogues on dopa decarboxylation by hog kidney enzyme. They found no inhibition of dopa decarboxylation by tryptophan itself. 
As far as we know, a competition of alternative substrates for hepatic AAD has not yet been described. In the present paper we therefore tried to check, if decarboxylation of phenylalanine, tyrosine or dopa in the liver may be influenced by tryptophan.

\section{Materials and methods}

Supernatant $20000 \mathrm{x} g$ of liver homogenate (1:10 in water) of Wistar male rats (VÚFB Konárovice) was used as the source of enzyme. The law on the keeping and care of laboratory animals was followed during treating the rats. The protein content was determined according to the method of Lowry (13).

Chemicals used in $A A D$ assay: $1-{ }^{14} \mathrm{C}$-labelled substrates: D,L-3-(3,4-dihydroxyphenyl)alanine-1-14 C (specific radioactivity 53.2 $\mathrm{uCi} \mathrm{mmol}^{-1}$, DuPont NEN, Germany), L-1${ }^{14} \mathrm{C}$-tyrosine (specific radioactivity $113.6 \mu \mathrm{Ci} . \mathrm{mmol}^{-1}$, Radiochemical Centre Amersham), and L-phenylalanine-1${ }^{14} \mathrm{C}$ (specific radioactivity $16.7 \mu \mathrm{Ci} . \mathrm{mmol}^{-1}$, Radiochemical Centre Amersham), L-tryptophan (LOBA Feinchemie, Austria), coenzyme: pyridoxal-5'-phosphate (Koch-Light Labs.), dioxane scintillation cocktail (Spolana, Neratovice). All chemicals used in AAD assays were at least of analytical grade.

$A A D$ assay and evaluation of the influence of tryptophan: The enzyme activity was determined radiometrically: The incubation mixture contained the liver preparation, pyridoxal-5'-phosphate $\left(1 \times 10^{-6} \mathrm{~mol}^{-1}\right.$ in most experiments), Ltryptophan $\left(1.0-3.0 \times 10^{-2} \mathrm{~mol}^{-1}\right)$ and an appropriate substrate: either $1{ }^{14} \mathrm{C}$-dihydroxyphenylalanine (final concentrations $1.0-9.0 \times 10^{-4} \mathrm{~mol}^{-1} \mathrm{l}^{-1}$, or ${ }^{1-{ }^{14}} \mathrm{C}$-tyrosine (final concentrations $1.1 \times 10^{-4}-1.0 \times 10^{-3} \mathrm{~mol}^{-1}$ ) or $1{ }^{14} \mathrm{C}$-phenylalanine (final concentrations $1.5 \times 10^{-4}-1.35 \times 10^{-3}$ mol. $1^{-1}$ ), in $0.02 \mathrm{M}$ Na-phosphate buffer, $\mathrm{pH}$ 6.8. Concentration of tryptophan used in the incubation mixture was set with regard to solubility of the amino acid and participation of other components of the mixture. Control samples were without tryptophan. The samples were then incubated under nitrogen at $37{ }^{\circ} \mathrm{C}$ for $30 \mathrm{~min}$ and the radioactivity of ${ }^{14} \mathrm{CO}_{2}$ (liberated from the mixture by means of sulfuric acid and absorbed in $0.1 \mathrm{ml} 30 \% \mathrm{KOH}$ ) was measured in the scintillation cocktail using 1219 Rackbeta scintillation counter LKB Wallac. Four samples were measured at individual concentrations of substrates. AAD activities were calculated on the basis of known amount and specific radioactivity of ${ }^{14} \mathrm{CO}_{2}$ produced in catalyzed reaction with individual substrates and expressed in nkat or pkat per $\mathrm{g}$ of protein as the means \pm S.D.

\section{Results and discussion}

The investigation of the influence of tryptophan on AAD showed that L-tryptophan is able to decrease decarboxylation of L-tyrosine, when concentration of tryptophan is by one order higher than that of tyrosine (Fig. 1). Line-
Fig. 1: Influence of tryptophan $\left(5.5 \times 10^{-3} \mathrm{M}\right)$ on decarboxylation of tyrosine by rat liver (supernatant $20.000 \mathrm{xg}$ of cell homogenate). The Michaelis-Menten plot is presented in the main graph; the insert shows the Lineweaver-Burk plot based on the same data. The assay was carried out at pH 6.8 (0.02 M sodium phosphate buffer).

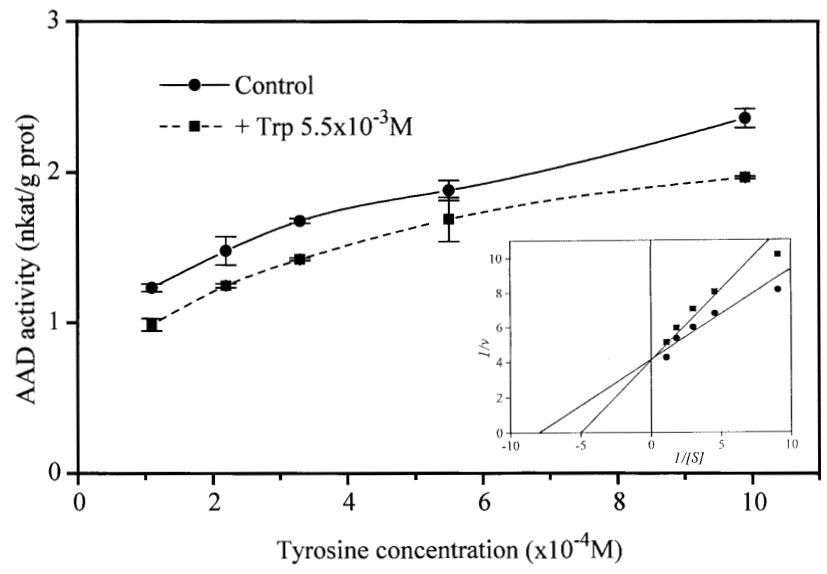

Fig. 2: Influence of tryptophan $\left(1.5 \times 10^{-2} \mathrm{M}\right)$ on decarboxylation of phenylalanine by rat liver (supernatant $20.000 \mathrm{xg}$ of cell homogenate). The Michaelis-Menten plot is presented in the main graph; the insert shows the LineweaverBurk plot based on the same data. The assay was carried out at pH 6.8 (0.02 M sodium phosphate buffer).

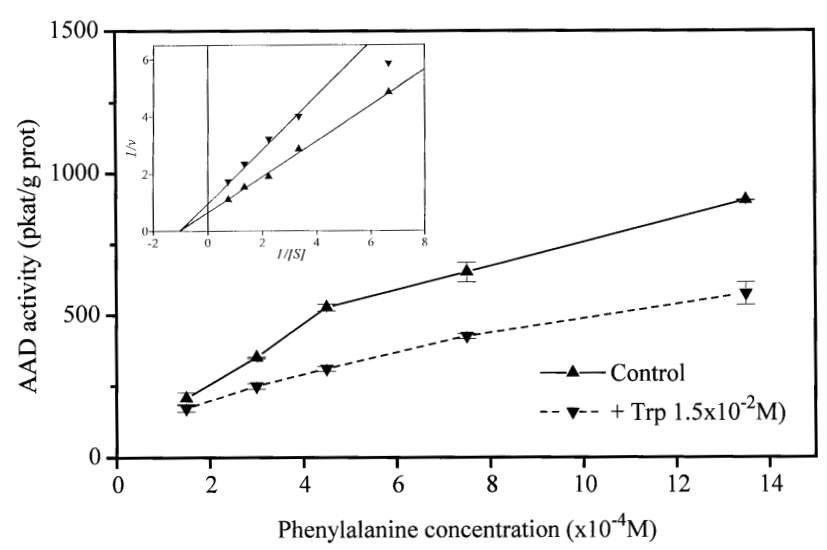

Tab. 1: Rat liver AAD activity with substrate DOPA in presence of tryptophan.

\begin{tabular}{|c|cc|}
\hline $\begin{array}{c}\text { DOPA } \\
\text { concentration } \\
\left(\mathrm{x} 10^{-4} \mathrm{M}\right)\end{array}$ & $\begin{array}{c}\text { AAD activity in nkat/g prot } \\
\text { Control } \\
(100 \%)\end{array}$ & $\begin{array}{c}\text { Tryptophan } \\
5 \times 10^{-3} \mathrm{M}\end{array}$ \\
\hline 1.0 & $10.26 \pm 0.06$ & $10.15 \pm 0.20(98.9 \%)$ \\
2.0 & $19.12 \pm 0.64$ & $18.98 \pm 0.27(99.2 \%)$ \\
3.0 & $29.31 \pm 1.01$ & $28.50 \pm 0.34(97.2 \%)$ \\
5.0 & $46.80 \pm 0.29$ & $43.97 \pm 0.01(94.0 \%)$ \\
9.0 & $59.75 \pm 0.13$ & $53.50 \pm 0.35(89.5 \%)$ \\
\hline
\end{tabular}


weaver-Burk double-reciprocal plot suggests a competition between both amino acids. If the effect of tryptophan is viewed in terms of inhibition, the constant of inhibition $K_{i}$ (determined graphically from the Lineweaver-Burk plot on the basis of the change of the slope, see the insert in Fig. 1) is close to $9.2 \times 10^{-3} \mathrm{M}$. The competitive character of inhibition of tyrosine decarboxylation by tryptophan is supported by the fact that no relevant influence was found, when we used lower concentrations of tryptophan (the results are not presented here). Our observation together with the findings of other authors, assessing the normal concentrations of tryptophan and tyrosine in blood plasma to be similar, i. e. about $50 \mu \mathrm{M}$ for tryptophan and $30 \mu \mathrm{M}$ for tyrosine (8) suggest that the decarboxylation of tyrosine in the liver is not influenced by tryptophan under physiological conditions.

In case phenylalanine was used as the AAD substrate, tryptophan inhibited decarboxylation as illustrated by Fig. 2. Lineweaver-Burk plot suggested formally non-competitive inhibition of phenylalanine decarboxylation caused by tryptophan, $K_{i}$ being about $2.75 \times 10^{2} \mathrm{M}$. In comparison, $K_{m}$ for L-phenylalanine decarboxylation, estimated from the same figure, is close to $1.2 \times 10^{3} \mathrm{M}$. Since the normal concentration of tryptophan in plasma is the same or even slightly lower than that of phenylalanine (8), it seems that high loads of tryptophan injected or given orally would be necessary to influence decarboxylation of phenylalanine in the liver.

As it was supposed, the influence of tryptophan on dopa decarboxylation was even much lower than on the decarboxylation of tyrosine and phenylalanine (for results see Table 1). Except high doses of L-DOPA (14), as in treatment of patients with parkinsonism, decarboxylation of 3 , 4-dihydroxyphenylalanine in the liver seems to be of negligible importance. Nevertheless, it is surprising that we have found some mild effect of tryptophan on dopa decarboxylation at all. The effect, expressed in \% of control AAD activity, was more pronounced with increasing concentration of dopa. We have no explanation for this apparent paradox. As the effect of tryptophan on decarboxylation of dopa was small, we have not succeeded to quantify it in terms of inhibition kinetics and inhibition constant.

\section{Conclusions}

1. Decarboxylation of phenylalanine and tyrosine in the liver is not influenced by tryptophan at a normal plasma concentration (about $50 \mu \mathrm{M}$ ) of this amino acid. The influence of large loads of tryptophan, which would increase the concentration of tryptophan by orders, is possible.

2. The effect of tryptophan on decarboxylation of 3, 4-dihydroxyphenylalanine is negligible and even large loads of tryptophan could not affect dopa decarboxylation efficiently.

\section{Acknowledgments}

This work was supported by the Czech National Research Council of Ministry of Health, Grant No. 4097-3. The authors wish to thank Prof. M. Lázníček (Radioisotope Laboratory, Charles University Faculty of Pharmacy) for the measurement of samples on a liquid scintillation counter and Mrs. B. Navrátilová for technical assistance.

\section{References}

1. Marklová E, Malina L, Hais IM. Urinary excretion of indolyl-acryloylglycine in some skin affections. Clin Chim Acta 1975;64:273-80

2. Marklová E. Where does indolylacrylic acid come from? Amino Acids 1999; 17:401-13

3. Holtz P, Heise R, Lüdtke K. Fermentativer Abbau von Dioxyphenylalanin durch Niere. Arch Exptl Pathol Pharmakol 1938;191:87-118.

4. Udenfriend S, Clark CT, Titus E. 5-Hydroxytryptophan decarboxylase: a new route of metabolism of tryptophan. J Am Chem Soc 1953;75:501-2.

5. Lovenberg W, Weissbach H, Udenfriend S. Aromatic L-amino acid decarboxylase. J Biol Chem 1962;237:89-92.

6. David JC. Tryptophan decarboxylation: a quantitatively significant route of tryptophan metabolism. FEBS Lett 1975;55:81-3.

7. Antonas KN, Coulson WF. Concentrations of phenylalanine and tyrosine in plas$\mathrm{ma}$ and brain of rats treated with phenylalanine loads. Biochem Soc Trans 1976;4:94-7.

8. Neckers LM, Delisi LE, Wyatt RJ. Liquid-chromatographic quantification of plasma phenylalanine, tyrosine, and tryptophan. Clin Chem 1981;27:146-8.

9. Dršata J, Hais IM. Aromatic amino acid decarboxylase activity of rat brain and its parts following exposure to ionizing radiation. Radiat Res 1974;59:724-6.

10. Dršata J, Ulrichová J, Walterová D. Inhibition of aromatic amino acid decarboxylase by sanguinarine. J Enz Inhibit 1996;10:231-7.

11. De Ropp RS, Furst A. Effect of analogues of phenylalanine and tryptophan on kinetics of dopa decarboxylase in rat brain. Brain Res 1966;2:323-32.

12. Bosin TR, Baldwin JR, Maickel RP. Inhibition of DOPA decarboxylation by analogues of tryptophan. Biochem Pharmacol 1978;27:1289-91.

13. Lowry OH, Rosebrough NJ, Farr AL, Randall RJ. Protein measurement with the Folin phenol reagent. J Biol Chem 1951;193:265-75.

14. Andersson I, Granerus AK, Jagenburg R, Svanborg A. Intestinal decarboxylation of orally administered L-dopa. Influence of pharmacological preparations, dose magnitude, dose sequence and food intake. Acta Med Scand 1975;198:415-20.

Submitted December 1999.

Accepted March 2000.

Prof. MUDr. Jaroslav Dršata, CSc., Charles University in Prague, Faculty of Pharmacy, Heyrovského 1203, 50005 Hradec Králové, Czech Republic. e-mail:drsata@faf.cuni.cz 